\title{
Are we doing enough to promote the effective use of mosquito repellents?
}

\section{Health authorities need to review recommendations on how to choose and use mosquito repellents}

$\mathrm{M}$ osquito-borne pathogens remain a threat to public health in Australia. The activity of dengue and chikungunya viruses has increased across South-East Asia and the Pacific region in recent years, and the number of travellers returning to Australia infected with mosquito-borne pathogens has steadily grown. ${ }^{1}$ Annual notifications of endemic mosquito-borne disease resulting from infection with Ross River or Barmah Forest viruses persist at around 5000 cases a year in Australia, and local transmission of dengue viruses remains a threat in Far North Queensland. $^{2}$

Notwithstanding the risk to human health, the nuisance biting of local mosquito species is also a problem. With the threat of exotic mosquito introduction, particularly of Aedes albopictus (Asian tiger mosquito), which could become established in major metropolitan regions, ${ }^{3}$ management of public health risks associated with mosquito populations will continue to be of concern.

\section{"the advice provided rarely reflects the wide range of commercially available mosquito repellent formulations"}

While broad-scale mosquito control can reduce the risk of mosquito-borne disease in certain circumstances, ${ }^{4}$ local authorities rarely have the financial or operational capacity to implement and maintain an effective program. New "technological fixes", such as the use of insect-specific intracellular bacteria to prevent mosquitoes transmitting dengue viruses, ${ }^{5}$ may assist in reducing disease risk, but these approaches are typically specific to mosquito species or pathogens.

Personal protection strategies, particularly the use of topical insect repellents, are therefore likely to remain the most widely encouraged strategy to reduce mosquito-borne disease risk.

\section{What do health authorities recommend?}

Recommendations on personal protection measures to avoid mosquito bites are provided in fact sheets and accompany seasonal warnings issued by a range of health authorities, including federal and state health departments and local governments. These recommendations usually include:
Cameron E Webb PhD, BSc(Hons)

Westmead Hospital and University of Sydney, Sydney, NSW.

cameron.webb@ health.nsw.gov.au

doi: 10.5694/mjal4.01237

Online first 09/02/15

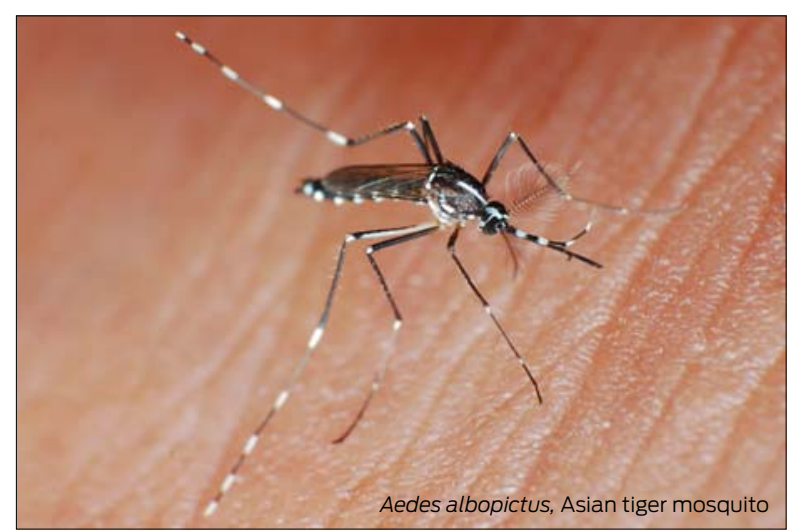

- use of insect repellents or insecticides (eg, topical repellents, mosquito coils)

- source reduction (eg, minimising opportunities for mosquitoes around dwellings by emptying or covering water-holding containers)

- behavioural practices (eg, avoiding mosquito habitats or times of the day when mosquitoes are most active)

- physical barriers (eg, bed nets, long-sleeved shirts). ${ }^{6}$

With regard to insect repellents, generally only products containing either $\mathrm{N}, \mathrm{N}$-diethyl-3methylbenzamide (commonly known as DEET) or 2-(2-hydroxyethyl)-1-piperidinecarboxylic acid 1-methylpropyl ester (commonly known as picaridin) are recommended. Published laboratory and fieldbased studies have shown DEET and picaridin to be the most effective mosquito repellents. ${ }^{7}$ These two products have also been shown to pose no substantial adverse health effects to users and are considered safe, ${ }^{8}$ although they are generally not recommended for children under 3 months of age. ${ }^{6}$ There should be no hesitation in recommending either of these products for use by older children or adults to protect against mosquito bites.

However, the advice provided rarely reflects the wide range of commercially available mosquito repellent formulations, how the concentrations of active ingredients in these products should be interpreted, or how they should be used.

\section{How can we compare repellents?}

All products marketed as mosquito repellents must be approved by the Australian Pesticides and Veterinary Medicines Authority (APVMA). There are currently more than 100 registered repellent formulations (including creams, gels, lotions, sprays, patches and wristbands). However, repellent formulation is 
generally far less important than the active ingredients contained therein. While most registered products contain DEET or picaridin, there are also a few formulations that contain botanical-based active ingredients.

In assessing repellents, both repellency (the percentage of mosquitoes landing on treated skin compared with untreated controls) and protection time (the period of time after application of a repellent during which no mosquito landings occur) are typically reported. Preventing, not just reducing, mosquito bites is critical in minimising mosquito-borne disease transmission. ${ }^{9}$ An understanding of how protection times differ between repellent formulations not only provides a guide to the most effective products but can also guide better communication of recommended use patterns, such as reapplication times.

Formulations containing DEET or picaridin can vary in the concentration of active ingredients. There is often a misconception that "stronger" formulations provide better protection by preventing more bites. However, the concentration of active ingredient determines how long a repellent provides protection, not the degree of protection that is provided. For example, "high-dose" and "low-dose" DEET-based repellents will provide comparable protection against biting mosquitoes up to about 2 hours, but the protection provided by the highdose repellent will persist beyond the time at which the low-dose repellent fails..$^{10}$

Repellents containing botanical extracts are often perceived as "safer" or "natural" alternatives to DEET or picaridin. The most common such active ingredients include Melaleuca and Eucalyptus oils. However, studies have shown that these repellents provide much shorter periods of protection against biting insects. ${ }^{11}$ By way of comparison, botanical-based repellent formulations would need to be reapplied three to four times more frequently than a $20 \%$ DEET- or picaridin-based repellent to provide comparable protection times. ${ }^{10}$ Botanical repellents are rarely recommended but given the interest in them in the community, an emphasis on their high required rate of reapplication may be needed.

A product often perceived to be a botanical repellent is $p$-menthane-3,8-diol (PMD). In Australia, PMD is registered with the APVMA as "Extract of lemon eucalyptus, being acid modified oil of lemon eucalyptus (Corymbia citriodora)". It is not an essential oil but rather a by-product of the hydrodistillation process. Laboratory and field tests have demonstrated that PMD provides effective protection against mosquito bites, ${ }^{12}$ but it is rarely included in lists of recommended repellents. This may be due to the relatively limited distribution of PMD products locally.

\section{Do we know how to effectively use repellents?}

Very rarely is advice provided on how repellents should be applied. This communication gap may contribute to deficiencies in the protection provided by even the most effective repellent formulations. Repellents, regardless of their active ingredient, should be applied as a thin, even coating on all exposed areas of skin. Spraying on clothes or a dab "here and there" will not provide complete protection. Reapplication after swimming and exercise is typically required.

There is a paucity of attitudinal and behavioural research into the use of topical insect repellents. Although instructions to "apply to exposed skin" are commonly included on repellent formulation labels, perhaps stronger advice should also accompany recommendations from local health authorities.

It is also important to consider other registered products among commercial mosquito repellents. Wristbands and patches purporting to provide protection against mosquitoes are registered, but studies have shown these "spatial" repellents to be ineffective. ${ }^{13}$ There is also an emerging range of cosmetic products combining mosquito repellents with sunscreens and skin moisturisers, which may require specific recommendations on use. ${ }^{14}$

Finally, different recommendations for repellent use will be required in regions where day-biting mosquitoes are present and pose a risk of dengue virus transmission (eg, Far North Queensland) compared with those needed elsewhere, where predominantly evening-biting mosquitoes are present. ${ }^{15}$

Topical mosquito repellents will remain a key component of recommended personal protection strategies against mosquitoes. When developing recommendations, it is important to consider not only the published scientific literature but also the current range of commercially available formulations, as this is likely to fuel concerns and drive enquiries from the public.

Ideally, nationally consistent guidelines would help local and state government health authorities develop strategies to promote the effective use of mosquito repellents to reduce nuisance biting and mosquitoborne disease risk.

Competing interests: My department, Pathology West, Institute for Clinical Pathology and Medical Research, Westmead, has received consultancy fees for laboratory testing of insect repellent formulations from companies and individuals associated with the development, manufacture, distribution and marketing of insect repellents in Australia.

Provenance: Not commissioned; externally peer reviewed. 
1 Knope K, National Arbovirus and Malaria Advisory Committee, Giele C. Increasing notifications of dengue in Australia related to overseas travel, 1991 to 2012. Commun Dis Intell Q Rep 2013; 37: E55-E59.

2 Russell RC, Kay BH. Medical entomology: changes in the spectrum of mosquito-borne disease in Australia and other vector threats and risks, 1972-2004. Aust J Entomol 2004; 43: 271-282. doi: 10.11ו1/j.1326-6756.2004.00436.x.

3 Russell RC, Williams CR, Sutherst RW, Ritchie SA. Aedes (Stegomyia) albopictus - a dengue threat for southern Australia? Commun Dis Intell Q Rep 2005; 29: 296-298.

4 Tomerini DM, Dale PE, Sipe N. Does mosquito control have an effect on mosquito-borne disease? The case of Ross River virus disease and mosquito management in Queensland, Australia. J Am Mosq Control Assoc 2011; 27: 39-44.

5 Moreira LA, Iturbe-Ormaetxe I, Jeffery JA, et al. A Wolbachia symbiont in Aedes aegypti limits infection with dengue, Chikungunya, and Plasmodium. Cell 2009; 139: 1268-1278.

6 NSW Health. Health warning on mosquito viruses. http:// www.health.nsw.gov.au/news/Pages/20140221_0.aspx (accessed Aug 2014).

7 Goodyer LI, Croft AM, Frances SP, et al. Expert review of the evidence base for arthropod bite avoidance. J Travel Med 2010; 17: 182-192.

8 Antwi FB, Shama LM, Peterson RK. Risk assessments for the insect repellents DEET and picaridin. Regul Toxicol Pharmacol 2008; 51: 31-36.
9 Debboun M, Strickman D. Insect repellents and associated personal protection for a reduction in human disease. Med Vet Entomol 2012; 27: 1-9.

10 Webb CE. Insect repellents derived from Australian plants and implications for public health messages. In: Debboun M, Frances SP, Strickman D, editors. Insect repellents handbook. 2nd ed. Boca Raton, Fl: CRC Press, 2014: 213-230.

1 Maguranyi SK, Webb CE, Mansfield S, Russell RC. Are commercially available essential oils from Australian native plants repellent to mosquitoes? J Am Mosq Control Assoc 2009; 25: 292-300.

12 Carroll SP, Loye J. PMD, a registered botanical mosquito repellent with DEET-like efficacy. J Am Mosq Control Assoc 2006; 22: 507-514.

13 Webb CE, Russell RC. Do wrist bands impregnated with botanical extracts assist in repelling mosquitoes? Gen Appl Entomol 2011; 40: 1-5. http://www.entsocnsw.org.au/ images/stories/media/40\%20webb\%20and\%20russell\%20 wrist\%20bands.pdf (accessed Nov 2014).

14 Webb CE, Russell RC. Insect repellents and sunscreen: implications for personal protection strategies against mosquito-borne disease. Aust N Z J Public Health 2009; 33: 485-490.

15 Webb CE, Russell RC. Advice to travellers on topical insect repellent use against dengue mosquitoes in Far North Queensland, Australia. J Travel Med 2011; 18: 282-283. . 